DOI: $10.2478 /$ jolace-2021-0012

\title{
Colour idioms in linguocultural comprehension of implicit meaning in the English and Azerbaijani languages
}

\author{
Pustakhanim Yusifova \\ Azerbaijan University of Languages in Baku, Azerbaijan \\ yusifo.pustekhanim@adu.edu.az
}

\begin{abstract}
The article deals with the comprehension of colour idioms in English and Azerbaijani in the linguacultural world. Above all, the paper covers the definition of idioms and various approaches by prominent English and Azerbaijani linguists; presents classification of idioms namely, semantically and structurally. Furthermore, syntactical study of constituents comprising colour idioms is being considered in the compared languages.

This article also discusses the implicit nature of colour idioms in English and Azerbaijani. As the title of the paper suggests, the term "implicit meaning" - the meaning that may not be obvious for the speakers of foreign languages - is also being considered in the article. The research work mainly analyses common colour idioms found in the English and Azerbaijani languages. The collected data are being studied on two aspects: analysis of colour idioms that coincides structurally and semantically both in English and Azerbaijani, as well as analysis of colour idioms that do not have semantic or structural equivalents in the compared languages.

Key words: color idioms, implicit meaning, compared languages, English, Azerbaijani.
\end{abstract}

\section{Introduction}

Idioms are internal part of the language and can be considered as linguistic universals. Every language has its own idioms, yet idioms with the same or similar meanings can be found in kindred and non-kindred languages. People from different languages and cultures may use either same or completely different combination of words to express the same meaning. Idioms or idiomatic expressions are the word combinations which don't make a sense when first read or heard. It is due to the fact that each language has its culture-specific idioms that may seem incomprehensible at first sight.

The term idiom comes from the Greek lexeme idios, which means "own, private, peculiar." This alone helps to uncover the true nature of the idioms. Idioms are implicit in character for the people belonging to different languages and cultures. Makkai identifies the term 'idiom' for "multiword expressions whose meaning is not predictable from their component parts" (Makkai, 1972, p. 122). Such kind of approach to the idioms can also be found in the works of Fraser (1970) and Fernando (1996). We think this is the best definition of the idioms and it was approved by almost all the scientists all over the world, as well as by the Azerbaijani linguists.

Oxford dictionary defines the word idiom as following: "Idiom is a group of words whose meaning is different from the meanings of the individual words" (Oxford Learner's Dictionary, 2021).

The detailed classification of idioms belongs to Fernando (Fernando, 1996, p. 35-36). According to him idioms can be pure idioms, semi-idioms, and literal idioms.

1) Pure idioms are non-literal multi-word idioms.

2) Semi-idioms are the idioms consisting of two constitutes, one with literal and the other with non-literal meaning. 
3) Literal idioms on the other hand are less semantically complex idioms compared with pure idioms and semi-idioms.

In the Azerbaijani language the idioms are classified based on the classification of idioms by well-known Russian linguist Vinogradov. Salim Jafarov identifies the following types of Azerbaijani idioms (Jafarov, 2007, p. 89-93):

1) Idiomatic junctions - the constituents of idiomatic junctions lose their literal meanings and together acquire a new meaning within a context. E.g. göz qoymaq.

2) Idiomatic unities: even if they remind idiomatic junctions for many aspects, the first word comprising the combinations can acquire affiliation suffix which is characteristic for the Azerbaijani language. E.g. Kallani yera qoymaq.

3) Idiomatic combinations: in idiomatic combinations the first element of the combination, on one hand, can take an affiliation suffix, on the other hand, idiomatic combinations can be split apart by other words. E.g. ürayindən keçmək - ürayindən çox şeylar keçmak.

English idioms can also be classified structurally. McCarthy and O'Dell (2002, p. 6) identify seven categories of idioms based on their structure.

1) Verb + object/complement: to kick the bucket.

2) Prepositional phrase: by brook or by hook

3) Compound: the other side of the coin

4) Simile (as + adj. + as): as mad as hatter

5) Binominal (word +and + word): black and white

6) Trinomial (word + word + and + word): cool, calm, and collected.

7) Whole clause or sentence: to cut the long story short.

Idioms are useful and comprehensible within the context as they are implicit in nature when used out of the context. However, sometimes even the context alone is not enough to decode the meaning of an idiom. To do so, one must have linguistic and cultural background of the foreign language.

Idioms are implicit in character for the speakers of the foreign languages as the constituents are not used in their direct meaning. When used in cross-cultural communications, the implicit nature of the idioms may cause misunderstanding and lead to pragmatic failure. According to Strasser "Idioms and their literal counterparts in isolation are made of the same logical presupposition and of a common assertion.... This property of idioms is often made use of the humorous effect, and is achieve humorous effects, and it is left up to the hearer to decode the complex meaning" (Strassler, 1982, p. 110).

But what is an implicit meaning?

Larson (1984) indicates implicit meaning as the meaning which is not shown but intended by the speaker and is a part of a communication process. Implicit meaning is not stated explicitly by lexical means or grammatical forms. Dienes and Perner (1998) define explicit vs implicit as following: "We ordinarily say that a fact is conveyed explicitly if it is expressed by the standard meaning of the words used. If something is conveyed but not explicitly, then we say it has been conveyed implicitly."

Larson classified implicit meaning into three types (Larson, 1998, p. 45): Implicit referential meaning. A speaker uses certain word or sentence in its denotational meaning; thus, the readers/hearers can understand what the word refers to using his/her background knowledge. Implicit situational meaning means understanding a word, phrase or a sentence taken from the context. Words, phrases or sentences may denote 
various meanings depending on the context and may be comprehended differently depending on the age, sex, cultural and linguistic background of the reader/listener. Implicit organizational meaning deals with the character of the information. Implicit organizational meaning is signaled by deictics, repetition, groupings and by many other features in the grammatical structure of a text (Falentina, 2015). The use and understanding of the idioms refer to implicit situational meaning.

\section{Problems of the study}

The following problems have been covered in the research paper:

1. What are the forms of the colour idioms in the English and Azerbaijani languages?

2. What are the implicit meanings of the colour idioms in the English and Azerbaijani languages?

3. Are there any identical colour idioms in the English and Azerbaijani languages?

\section{Aims of the study}

The aims of the study include analysing structure of the colour idioms; identifying implicit meanings of the colour idioms in English and Azerbaijani; as well as defining colour idioms of the same kind in the English and Azerbaijani languages.

\section{Research method}

\subsection{Data source}

The data sources of the research paper were taken from dictionary of idioms in the English and Azerbaijani languages mentioned in the References section. Qualitative data was used throughout the paper i.e. colour idioms in English and Azerbaijani comprise the core of the research.

\subsection{Method and technique of collecting data}

The method of collecting data includes comparative, descriptive comparison, context analysis methods to analyse the collected data. Comparative method was used to find out similar and different colour idioms in the compared languages. Descriptive comparison method served to investigate the similar and different structures and semantics of colour idioms, as well as, how the idioms of the same constituents can have implicit character in linguocultural comprehension. Context analysis was utilised for defining constituent parts of the colour idioms with the identical and different meanings.

\section{Analysis of implicit nature of colour idioms in the English and Azerbaijani languages}

The colour idioms in English and Azerbaijani, on one hand may overlap structurally and semantically, and on the other hand they may lack equivalents in the foreign language. In this paper we are going to analyse either idioms.

\section{Analysis of sample data 1}

"A white lie (ağ yalan)" is an idiom existing both in English and in Azerbaijani. The idioms in both languages have the structure of adjective + noun, consist of the same words, and the second constituent of the idiom has preserved its referential meaning. White lie in English means "an innocent lie, a lie told to save someone from embarrassment", whereas in Azerbaijani ăg yalan is used in the meaning of "a complete lie." As seen from the given samples, this idiom carries different meanings in compared languages that may be illegible to the speakers of different languages. 


\section{Analysis of sample data 2}

"In Black and white (ağ-qara)" is also one of the idioms that can be found in both languages. This idiom has a structure of adjective +(and) + adjective in English and Azerbaijani. "In" is a preposition and is used only in English version of the idiom as prepositions are not characteristics for the Azerbaijani language. Moreover, the idiom "Black and white" in English and Azerbaijani has the same meaning - the meaning of "words in print or on paper". Thus, this idiom being explicit in character is unlikely to cause pragmatic failure between people belonging to two different cultures and languages.

\section{Analysis of sample data 3}

"Black Friday" is an idiom consisting of an adjective + noun. Any reader would take this idiom for something "bad or unfortunate", however, this idiom is used to denote Friday following the Thanksgiving Day in the USA. Many stores offer sales on this particular day, so in reality this idiom is not associated with bad or unfortunate as one may think. The Azerbaijani counterpart of this idiom is "Qara cümə" which has become popular only in recent years. This idiom is also related to the day of great sales. The Azerbaijani idiom possesses the identical structure, comprising of an adjective (qara-black) + noun (cüməFriday).

\section{Analysis of sample data 4}

The idiom "Black market (qara bazar)" (Veliyeva, 2010, p. 176) is also one of the colour idioms existing both in English and in Azerbaijani. Structurally, the idiom consists of adjective + noun. The constituent parts of the idiom are comprised of the same words - the first word adjective denoting colour and the second a noun denoting "market" for the English and Azerbaijani languages. Even if the idiom seems explicit at first glance, it has an implicit meaning of "an illegal traffic or trade in officially controlled or scarce commodities."

\section{Analysis of sample data 5}

"Black humour" and its Azerbaijani correlative "Qara yumor" (Veliyeva, 2006, p. 284) have the identical structure and meaning. Structurally, these idioms are composed of adjective (black-qara) + noun (humour-yumor). As the word "black" is associated mainly with "bad or unfortunate" both in English and Azerbaijani languages and cultures, the word preserves its actual sense within the given idioms. Semantically, the idioms overlap, meaning "a humorous way of looking at or treating something that is serious or sad."

\section{Analysis of sample data 6}

The idiom "Once in a blue moon (Qırmızı qar yaganda)" is completely different both in structure and in constituent words in English and Azerbaijani. Despite answering to the question when? in both languages, in English the idiom is a noun phrase (NP), however, in Azerbaijani it is a verb phrase (VP). In Azerbaijani the meaning of immediate constituents is as following: "qırmızı" - red, "qar" - snow, "yağanda" - while snowing, and the whole idiom can be translated as "while it is snowing a red snow." Nevertheless, this idiom has the same meaning denoting "very rarely." As one can see from the structure and the constituents comprising the idiom, the idiom may seem implicit to the speakers of the English and Azerbaijani languages. 


\section{Analysis of sample data 7}

"Black sheep (ala qarğa)" is an idiom meaning "a person who is a disgrace to the family or to the group." The idiom in both languages possesses the structure of adjective + noun. Nonetheless, in Azerbaijani ala means "mixture of grey with other colours", and qarğa means "a crow." The first word of the idiom denotes colour and the second word denotes certain animal in the English and Azerbaijani languages. This idiom being implicit at first sight within a context may lead to misunderstanding between speakers of the target languages.

\section{Analysis of sample data 8}

In the Azerbaijani language there is an idiom "Qara camaat" (Veliyeva, 2010, p. 177). Literal translation of the idiom will be as "black people", however, such kind of idiom does not exist in the English language. This idiom has the structure of adjective + noun. The actual meaning being "lower class of the people, common people", is likely to carry an implicit nature for the speakers of English, because it lacks its English equivalent.

\section{Analysis of sample data 9}

The structure of the idiom "Qara geymək"(Veliyeva, 2010, p. 77) in Azerbaijani consists of adjective + verb. The literal translation of the idiom into English will be as "to wear black" implicitly meaning "to be in mourning or to mourn."

\section{Analysis of sample data 10}

The idiom "Qara yaxmaq" (Veliyeva, 2010: 180) also has a verbal structure (VP). The immediate constituents consist of an adjective and a verb. The word by word translation of the idiom into English is "to smear black." However, English equivalent of the idiom is "to throw mud at someone."

\section{Analysis of sample data 11}

"To feel blue" (Veliyeva, 2010, p. 164) is an idiom being verbal in nature, consists of infinitive + adjective. The second element of the idiom denotes colour. The Azerbaijani equivalent of the idiom is "Qanı qaralmaq." This idiom is verbal in nature, as well. The structure of the idiom is noun (blood) + deadjectival verb (to blacken). The second element also denotes colour in Azerbaijani. These idioms may seem implicit in structure for the speakers of the target languages, nevertheless, both idioms denote "to be disappointed, to feel sad or depressed" in the English and Azerbaijani languages.

\section{Analysis of sample data 12}

"Sarı mətbuat" (Veliyeva, 2010, p. 557) is an idiom in Azerbaijani having the structure of an adjectiv phrase (AP). The words constituting the idiom are adjective (sarl-yellow) + noun (matbuat - press). This idiom has no English equivalents but it means "gutter press." Thus, this idiom can be considered both structurally and semantically implicit.

\section{Analysis of sample data 13}

The idiom "Ağ gün" (Veliyeva, 2006, p. 23) consists of adjective + noun, "ağ" standing for white and "gün" standing for the day. The first word of the idiom denotes colour. There is no equivalent of the idiom in the English language. The idiom implicitly symbolizes "a happy life." 


\section{Analysis of sample data 14}

The idiom "Boz adam" (Veliyeva, 2006, p. 110) consists of adjective + noun. The first word "boz" means "grey" and denotes colour. The second word "adam" means "a person" in English. Not having an English analogue, the whole idiom is implicitly used in the sense of "a rude person." The second constituent of the idiom has preserved its actual meaning.

\section{Analysis of sample data 15}

"A black economy" and its Azerbaijani counterpart "qara iqtisadiyyat" (illegal economy) (Mustafayeva, 2008, p. 71) possess the same structure of adjective + noun. The words comprising the idiom are also the same, black stands for "qara" and economy stands for "iqtisadiyyat" in the Azerbaijani language. Even if at first sight these idioms seem implicit, they express "illegal economy." The second word of the idioms is used in its denotational meaning.

\section{Analysis of sample data 16}

"To give someone a red-carpet treatment" (Mustafayeva, 2008, p. 3) is an English idiom with verbal structure consisting of a verb (infinitive) + two complements. The second complement is modified by an adjectival phrase. This idiom means "to greet someone in the best way possible." The Azerbaijani correlative consists of completely different words. "Ayağının altında qurban kəsmək." "Ayaq" stands for foot, "altında" stands for under, and "qurban kəsmək" stands for to sacrifice in the English language. Thus, in Azerbaijani this idiom looks like "To sacrifice under one's feet" and this idiom does not involve any words denoting colour. However, idioms both in English and in Azerbaijani have the same structure of verb phrase. They are alike semantically, as well.

\section{Analysis of sample data 17}

"To be in the red" ("əli aşağl olmaq") (Mustafayeva, 2008, p. 75) are the idioms possessing a verbal structure. The constituents comprising the idiom "Oli aşağı olmaq" in the Azerbaijani language consists of the words "əli" (hand, "I" is an affiliation suffix), "aşağı" (low), and "olmaq" (to be). This idiom can be understood as "to have a lower hand." It is also important to mention that the Azerbaijani version of the idiom does not contain a word denoting "colour." Both of the idioms denote "to be short of money" in the compared languages.

\section{Analysis of sample data 18}

"To talk a blue streak" is a verbal idiom structurally consisting of an infinitive + a noun modified by an adjective. The adjective "blue" denotes colour and this idiom is used in the sense of "speak quickly and at length." The Azerbaijani counterpart of the idiom is "Dil otu yemək." (Gurbanova, 2017, p. 59) Although this idiom is also structurally verbal consisting of noun (dil-tongue) + noun (ot-grass, "- $u$ " is an affiliation suffix) + infinitive (yemak - to eat) meaning "to eat a tongue grass." This idiom is completely implicit and senseless for the speakers of the English language. Moreover, the Azerbaijani equivalent does not contain a word denoting colour.

\section{Analysis of Sample Data 19}

"Qırzmızı-qurmız" (Veliyeva, 2010, p. 226) is an Azerbaijani colour idiom consisting of a repetition of the word "qurmız" which stands for "red" in English. Structurally, the Azerbaijani idiom "qırmızı-qırmızı" is comprised of adjective + adjective. Word by word 
translation on the idiom into English will be "red-red" which does not make any sense. Thus, the idiom is completely implicit for the speakers of the English language. The actual meaning of the idiom is "with no sign of shame" and lacks its English counterpart.

\section{Analysis of Sample Data 20}

"To have a green thumb" (Gurbanova, 2017, p. 49) is an English idiom that has a verbal character. Structurally, this idiom consists of an infinitive (to have) + complement (thumb). The complement is modified by an adjective denoting colour. Semantically, the idiom stands for "to be good at gardening." The presented idiom lacks its Azerbaijani equivalent and the literal meanings of constituent parts may seem implicit and cause misunderstanding for the Azerbaijani speakers.

\section{Conclusion}

All in all, the research paper proposes thorough explanation of common English and Azerbaijani colour idioms and their implicit nature.

English and Azerbaijani colour idioms have different forms, however, adjective + noun structure prevails both in the English and in Azerbaijani languages. The compared idioms contain the following colour words: "black, white, red, yellow, blue, and green." The study also presents the meaning, composition, and the structure of the common colour idioms in the English and Azerbaijani languages. Notwithstanding a number of structurally and semantically correlating colour idioms in English and Azerbaijani, there are also variety of colour idioms without their corresponding counterparts in the compared languages which are most likely to be implicit for the speakers of the foreign languages. Even if one knows the meanings of the individual words comprising different idioms, one will still need a dictionary to refer to comprehend the meaning of the idioms. Moreover, sometimes English or Azerbaijani counterparts of the colour idioms may not contain words denoting colour.

\section{References}

Amir, S. (2012). Translation of Idioms and Fixed Expressions: Strategies and Difficulties. Theory and Practice in Language Studies, 2(6). doi: 10.4304/TPLS.2.6.1220-1229

Dienes, Z. \& Perner, J. (1999). A Theory of Implicit and explicit Knowledge. Behavioral and Brain Sciences, 22, 735-808. doi: https://doi.org/10.1017/S0140525X99002186

Falentina, C. (2015). The translation of implicit meaning in eldest novel. Available on: $\quad$ https://text-id.123dok.com/document/ky6epjloz-implicit-organizationalmeaning-implicit-meaning.html

Fernando, C. (1996). Idioms and Idiomacity. Oxford: OUP.

Gurbanova, F. (2017). Learn idioms through reading. Baku: Science and Education.

Jafarov, S. (2007). Contemporary Azerbaijani Language: Lexicology, vol. II. Baku: EastWest.

Larson, M. (1998). Meaning-Based Translation: A Guide to Cross-Language Equivalence. University Press of America.

Makkai, A. (1972). Idiom structure in English. The Hague: Mouton.

McCarthy, M. \& O'Dell, F. (2002). English Idioms in Use. Cambridge: Cambridge University Press.

Mustafayeva, S. (2008). Everyday English idioms with Azerbaijani Equivalents in use. Baku: Letterpress.

Oxford Learner's Dictionary. (2021). Available on: https://www.oxfordlearnersdictionaries.com/definition/english/idiom. 
Seidl, J. \& McMordie, W. (1988). English Idioms. $5^{\text {th }}$ edition. Oxford University Press.

Strassler, J. (1982). Idioms in English: A Pragmatic Analysis. Tübingen: Gunter Varr Verlag. Veliyeva, N. (2010). Azerbaijani-English-Russian Phraseological Dictionary, vol. II. Azerbaijan State Publishing House.

Veliyeva, N. (2006). Azerbaijani-English-Russian Phraseological Dictionary. Baku: Nurlan. Yusifova, P. (2018). Three Layers of Pragmatic Failure Across Languages and Cultures. International Journal of English Linguistics, 8(6). doi: 10.5539/ijel.v8n6p256

\section{Contacts:}

Pustakhanim Yusifova

Azerbaijan University of Languages

Baku

Azerbaijan

https://orcid.org/0000-0002-7156-8889

yusifo.pustekhanim@adu.edu.az 